\title{
THE OAO-B TELESCOPE
}

\section{A. BOGGESS III}

National Aeronautics and Space Administration, Goddard Space Flight Center, Greenbelt, Md., U.S.A.

The third Orbiting Astronomical Observatory will be launched in the Fall of 1970 carrying a $92 \mathrm{~cm}$ telescope and a scanning spectrometer. The instrument can produce stellar spectra in the range from 1000 to $4000 \AA$ at resolutions of 2,8 or $64 \AA$. Several entrance apertures can be used, including a $5^{\prime} \times 6^{\prime}$ slot, a $1^{\prime}$ circle and two narrow slits for special conditions. The spectrometer employs six pulse counting detectors spaced at $500 \AA$ intervals, and can also be used as a fixed wavelength six channel photometer for faint objects. The detection and data handling systems are designed to yield photometric data at a constant statistical precision of $1.6 \%$ with a maximum integration time of $42 \mathrm{~s}$ at each wavelength.

\section{DISCUSSION}

A. Monfils: The interval between steps is equal to the slit width. Why did you not choose half this interval?

A. Boggess: The original intent was to have one Ångström grating steps. Unfortunately, we were not able to build a reliable stepping mechanism for the small angle required.

$D$. D. Clark: Have you done any experiments to test the baffle system's ability to exclude sunlight?

A. Boggess: No, we have not the facilities for that test. Information from other satellites indicates that instrumental scattering from the daylit Earth will be the most severe problem, and that sunlight scattered from material in the vicinity of the spacecraft should be negligible.

$R$. J. Van Duinen: Could you say something about the baffle construction in view of daylight observations?

A. Boggess: Since the front end of the tube is cut to permit looking towards the Sun, it becomes particularly sensitive to scattered earthlight and this earthlight cannot be completely baffled out. The internal baffles are angled to reflect most of this light back out of the tube.

Note added in proof. The OAO-B satellite was launched from Cape Kennedy, Florida on November 30, 1970. Unfortunately, a nose fairing did not release and the satellite never achieved orbital velocity. Although a spare telescope and spectrometer exist, at present there are no plans to fly them on a future satellite. 